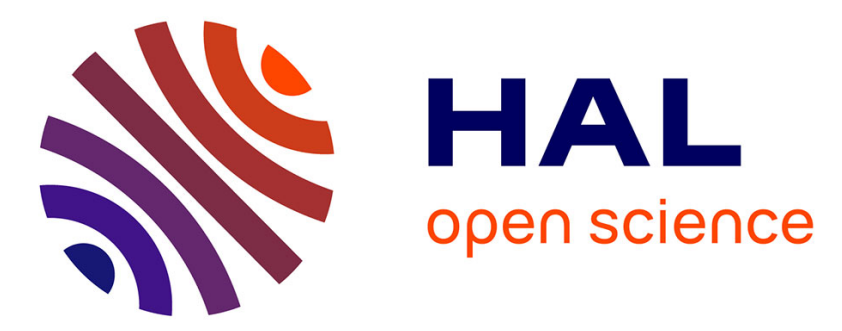

\title{
"Crise locale, crises nationales: rythmes et limites de la crise de la fin du Moyen Âge à Paris au miroir des prix fonciers"
}

Boris Bove

\section{- To cite this version:}

Boris Bove. "Crise locale, crises nationales: rythmes et limites de la crise de la fin du Moyen Âge à Paris au miroir des prix fonciers". Histoire urbaine, 2012, Villes en crise au Moyen Âge, pp.81-106. halshs-01465602

\author{
HAL Id: halshs-01465602 \\ https://shs.hal.science/halshs-01465602
}

Submitted on 12 Feb 2017

HAL is a multi-disciplinary open access archive for the deposit and dissemination of scientific research documents, whether they are published or not. The documents may come from teaching and research institutions in France or abroad, or from public or private research centers.
L'archive ouverte pluridisciplinaire HAL, est destinée au dépôt et à la diffusion de documents scientifiques de niveau recherche, publiés ou non, émanant des établissements d'enseignement et de recherche français ou étrangers, des laboratoires publics ou privés. 


\section{Boris BOVE, « Crise locale, crises nationales : rythmes et limites de la crise de la fin du Moyen Âge à Paris au miroir des prix fonciers ", Histoire Urbaine, $\mathrm{n}^{\circ} 33$ (2012), p. 81-106}

La notion de crise offre l'avantage, par sa généralité, de pouvoir s'appliquer à tous les domaines de la vie sociale. Cette vertu est d'autant plus précieuse qu'une crise dans un champ d'activité peut avoir des répercussions dans d'autres, voire, si on en croit l'historiographie de « la crise de la fin du Moyen Âge », agréger des facteurs aussi divers que le surpeuplement, les épidémies, l'économie seigneuriale, la guerre et l'impôt. Pensée à partir des blocages de l'économie rurale, la crise de la fin du Moyen Âge touche également les villes, et notamment Paris qui, par son statut de grande ville et de capitale, peut en effet être considéré comme l'épicentre de cette crise : la guerre frappe à ses portes dès 1346, la peste y fait des ravages en 1348, ses habitants se révoltent contre la mauvaise gestion de l'impôt à l'occasion des états généraux de 1355-1358, puis à nouveau en 1382-1384. La capitale est le cadre privilégié de la rivalité des ducs pour la tutelle du roi fou entre 1407 et 1418, avant d'être occupée par les Anglais en 1420 et décimée par les épidémies ou les famines dans les années suivantes. En 1437, Charles VII fait son entrée dans une ville ruinée, où les rois ne résideront plus jusqu'à François $\mathrm{I}^{\mathrm{er}}$. Le cas parisien devrait donc être un cas d'école illustrant les mécanismes de la crise des $\mathrm{XIV}^{\mathrm{e}}$ et $\mathrm{XV}^{\mathrm{e}}$ siècles. Pourtant cette impression de crise permanente est en contradiction avec la perception que les historiens peuvent avoir de la réalité économique de la ville jusqu'au début du $\mathrm{XV}^{\mathrm{e}}$ siècle : l'économie parisienne semble en effet plutôt bien résister aux difficultés de la conjoncture au XIVe siècle.

On voudrait donc souligner ici ce décalage en rappelant brièvement le mécanisme général de la crise, tel qu'il a été mis à jour, pour le confronter à des indicateurs socioéconomiques locaux que sont la démographie et surtout les prix de l'immobilier. Les transactions foncières sont une des rares sources sérielles permettant d'objectiver les fluctuations économiques dans la mesure où elles reflètent la solvabilité de la population tout en étant au cœur d'un système de crédit massivement utilisé par les citadins. Or ces indicateurs résistent bien au $\mathrm{XIV}^{\mathrm{e}}$ siècle ; lorsqu'ils virent au rouge dans les années 1420, crise immobilière et crise financière vont alors de paire, mais s'inscrivent dans un contexte plus vaste dont elles ne sont, en l'occurrence, que le reflet.

\section{La crise de la fin du Moyen Âge}

Les études de démographie historique des années 1960 ont permis de mettre à jour l'importance de la saignée opérée dans la population des villes et des campagnes par la peste noire de 1347, et sa résurgence régulière à partir de 1362. S'en est suivi dans les années 19701980 un débat entre historiens d'inspiration néomalthusienne et marxiste pour comprendre les cause de ce phénomène ${ }^{1}$. La controverse est depuis retombée et le débat semble tranché au profit des thèses marxistes, qui ont, du reste, prolongé plus que contredit les thèses néomalthusiennes ${ }^{2}$.

\footnotetext{
${ }^{1}$ Trevor Henry Aston, Charles Harding English Philpin (ed.), The Brenner Debate. Agrarian Class Structure and Economic Development in Pre-industrial Europe, London-New York-Melbourne, Cambridge university press, 1985.

${ }^{2}$ Pour une synthèse de ces débats, Boris Bove, Le temps de la Guerre de Cent ans, Paris, Belin, 2009, chap. XIV.
} 
De tout cela il ressort que la crise de la fin du Moyen Âge procède du décalage entre une croissance démographique soutenue et les ressources agricoles de plus en plus limitées. La crise est donc avant tout agraire et trouve son origine dans l'incapacité de la paysannerie à augmenter la production céréalière autrement que par l'extension de la surface agricole cultivée. Lorsqu'il n'y a plus rien à défricher, c'est la famine, qui réapparaît dès 1315. Les historiens néo-malthusiens interprètent l'incapacité des paysans à intensifier la production agricole comme un problème technique, tandis que les historiens d'inspiration marxiste incriminent l'organisation socio-économique. Pour eux, la rente seigneuriale enrichit le seigneur, mais le désintéresse de la production, tandis que les producteurs, faute de réserves financières, n'ont pas les moyens de risquer des innovations susceptibles d'intensifier la production. Se met alors en place le cercle vicieux de la dépression de la fin du Moyen Âge ${ }^{3}$.

En effet, tant qu'il y a croissance démographique, le nombre de tenanciers s'accroît et les revenus du seigneur restent stables, car il compense par des réductions de taxes les rendements décroissants des terres défrichées ; quand la croissance se stabilise faute de terres à défricher, les paysans sont incapables de produire assez pour nourrir leurs enfants, tandis que les revenus du seigneur baissent du fait de l'inflation. Le féodalisme est alors dans une impasse. L'état sanitaire dégradé des populations rurales fait ensuite le lit des épidémies à partir de 1347. Par ailleurs, les petits seigneurs cherchent à récupérer par d'autres moyens ce que l'évolution économique leur a fait perdre, le plus simple étant pour eux le service armé du roi qui leur permet de toucher une solde, ainsi que de piller et de rançonner l'adversaire. Dans cette perspective, la guerre apparaît comme le moyen pour l'aristocratie de développer une fiscalité royale qui lui profite directement et qui a l'avantage d'élargir son prélèvement aux villes dont la richesse échappait jusque-là aux nobles. La crise du féodalisme susciterait donc la résurgence de la peste noire, la guerre de Cent Ans et le développement de l'État. La guerre achève d'épuiser l'économie rurale en entraînant un sur-prélèvement fiscal sur les tenanciers. La désorganisation économique entraîne celle de la société et une chute démographique sans précédent, qui est aussi celle des revenus seigneuriaux. Se met ainsi en place un cercle vicieux qui se dénoue lorsque le retour à la paix permet aux paysans de remettre en valeur les bonnes terres laissées en friches par la disparition de leurs anciens tenanciers.

Ce schéma d'interprétation de la crise se fonde avant tout sur une analyse de l'économie rurale et des rapports sociaux dans les campagnes, mais les villes y ont leur place, bien qu'elles ne soient pas au cœur de la démonstration : leur densité démographique les rend plus vulnérables aux épidémies, l'insécurité entraînée par la guerre entrave la circulation des biens, la guerre leur coûte cher en soldes et en fortifications, d'autant que la taxation des transactions commerciales (l'aide) apparaît comme le moyen le plus simple de financer la guerre, mais frappe plus durement les citadins qui ne peuvent vivre en autarcie comme les paysans. Les nombreuses révoltes urbaines, en particulier à Paris, sont là pour prouver que les villes ont aussi été frappées par la crise de la fin du Moyen Âge qui, bien que d'origine agraire, s'étend à l'économie tout entière, à la démographie et à la politique.

Reste à prendre la mesure et les dimensions précises de cette crise en ville, et à Paris en particulier. Divers indices socio-économiques invitent à corriger pour le $\mathrm{XIV}^{\mathrm{e}}$ siècle l'image sinistre évoquée en introduction.

\footnotetext{
${ }^{3}$ Guy Bois, Crise du féodalisme. Economie rurale en Normandie du début du XIV siècle au milieu du XVI siècle, 1976, rééd. Paris, Presses de la Fondation nationale des sciences politiques, 1981 et id., La grande dépression médiévale, $X I V^{e}-X V^{e}$ siècles. Le précédent d'une crise systémique, Paris, PUF, 2000.
} 


\section{Relativité de la crise à Paris au XIVe siècle}

Mesurer la crise suppose de fonder l'analyse sur des indicateurs pertinents. Une méthode pour tenter de cerner l'impact de la crise sur la ville pourrait être de cerner l'évolution de sa population, puisqu'on peut partir du principe que la dépopulation est signe de dévitalisation de l'économie urbaine.

On ne dispose malheureusement pas de sources fiscales permettant de suivre l'évolution de la population urbaine après 1328 , mais on peut tenter de juger le dynamisme démographique de la ville à sa capacité à conserver ses faubourgs dans le contexte difficile de la fin du Moyen Âge. Beaucoup de villes voient en effet les leurs se vider avec la crise démographique et l'insécurité suscitée par la guerre de Cent ans - ainsi ceux de Saint-Flour, qui abritaient $45 \%$ de la population contribuable en 1345, n'en recensent que $8 \%$ en 1364 . Paris ne paraît pas épargnée par la peste, qui frappe aussi durement la capitale que les autres villes du royaume en 1348-1349, si bien qu'on a pu estimer à un tiers la chute de la population à ce moment ${ }^{4}$. Dans la ville neuve du Temple, peuplée de modestes artisans, les trous opérés par l'épidémie dans les classes laborieuses se traduisent par l'abandon de maisons ${ }^{5}$. La capitale est donc au diapason de la crise générale.

Pourtant ses faubourgs semblent avoir plutôt bien résisté à la saignée épidémique, comme le suggère le tracé de la nouvelle enceinte du XIV siècle (carte 1). Sa construction est ordonnée en 1356 dans l'urgence par le prévôt des marchands Étienne Marcel, comme une mesure d'auto-défense alors que tous s'attendent à une recrudescence des chevauchées anglaises après la capture du roi Jean le Bon à Poitiers en septembre ${ }^{6}$. Si on suit la chronique dite de Jean de Venette, « ils firent creuser des fossés au pied de la muraille côté ouest (sic) et autour des faubourgs côté est (sic), là où il n'y en avait jamais eu " ${ }^{7}$, c'est-à-dire que sur la rive gauche, la vieille enceinte de Philippe Auguste fut dotée d'un fossé et les déblais jetés derrière la muraille du XIII ${ }^{\mathrm{e}}$ siècle, mais que sur la rive droite, on fit creuser une nouvelle enceinte de $400 \mathrm{~m}$ en avant de l'ancienne. Le choix du tracé obéit donc explicitement au désir de protéger la population des faubourgs nord, huit ans seulement après que la peste avait saigné la ville. Il faut donc supposer que les pertes occasionnées par la peste ont été rapidement compensées par l'immigration, ce qui est probable vu les fortes densités de population en Île-de-France et l'attractivité économique de la ville ${ }^{8}$. Plus remarquable encore, ce tracé est maintenu dans le dernier tiers du $\mathrm{XIV}^{\mathrm{e}}$ siècle, alors même que les travaux de fortifications reprennent sur une base nouvelle: les fouilles du Carrousel du Louvre ont montré que le fossé d'Etienne Marcel est alors comblé et le nouveau fossé déplacé vers l'ouest d'une trentaine de mètres ${ }^{9}$. Ce n'est donc pas l'inertie qui pousse les bâtisseurs à maintenir ce tracé, mais bien la même volonté d'inclure les faubourgs dans la ville. Le nouveau périmètre de la ville incorpore certes des espaces vides, mais cela s'explique par des

\footnotetext{
${ }^{4}$ Michel Mollat, « Notes sur la mortalité à Paris au temps de la peste noire d'après les comptes de l'œuvre de Saint-Germain-1’Auxerrois », Le Moyen Âge, n 69, 1963, p. 505-527.

${ }^{5}$ Geneviève Etienne, Étude topographique sur les possessions de la maison du Temple à Paris (XII ${ }^{e}-X I V^{e}$ siècle), thèse manuscrite de l'ENC, p. 410.

${ }^{6}$ Henri Sauval, Histoire et recherche des antiquités de la ville de Paris, Paris, Charles Moette, 1724, I, p. 38 et III, 124. Albert Rigaudière, «Le financement des fortifications urbaines en France du milieu du XIV siècle à la fin du XV siècle », Revue historique, $\mathrm{n}^{\circ}$ 553, 1985, p. 19-22.

${ }^{7}$ Chronique dite de Jean de Venette, édité par Colette Beaune, Paris, Le Livre de Poche, 2011, p. 153.

${ }^{8}$ Alors que Ferdinand Lot constatait une densité moyenne de 7,7 feux au $\mathrm{km}^{2}$ en 1328, Guy Fourquin a pu prouver que la densité moyenne dans la prévôté de Paris est de 14 feux au $\mathrm{km}^{2}$ et que la riche plaine limoneuse de «France» permettait de faire vivre jusqu'à 19 feux au $\mathrm{km}^{2}$ (« La population de la région parisienne aux environs de $1328 »$, Le Moyen âge, $\mathrm{n}^{\circ}$ 62, 1956, p. 63-91).

9 Paul Van Ossel (sous la direction de), Les jardins du Carrousel (Paris). De la campagne à la ville: la formation d'un espace urbain, Paris, DAF, 1998, p. 149, 185-186.
} 
contraintes techniques : l'urbanisation s'est faite en étoile le long des principales rues qui rayonnaient à partir du centre ${ }^{10}$, mais cette forme en doigts de main est incompatible avec celle d'une enceinte annulaire, d'où des vides interstitiels. D'autre part, ces terrées qui établissent une défense en profondeur sur $90 \mathrm{~m}$ supposaient de nombreuses expropriations qu'un tracé en zone urbaine aurait compliqué, d'où le choix de passer, à Paris comme ailleurs ${ }^{11}$, dans des zones non-bâties. Enfin, l'inclusion de la culture Sainte-Catherine, peu urbanisée, s'explique par le souci d'inscrire le tracé du nouveau fossé dans la dépression marécageuse laissée par l'ancien bras mort de la Seine, tant pour faciliter sa mise en eau, que pour assainir la zone (carte 1) ${ }^{12}$. Ces contraintes techniques expliquent donc que la nouvelle enceinte englobe les terres non bâties, mais son tracé visait avant tout à protéger les zones urbanisées. Les faubourgs septentrionaux gardent donc leur importance à la fin du XIV siècle, en dépit du retour de la peste en 1362. Le cas parisien n'est pas unique, puisque parmi les villes qui se dotent de fortifications après 1347, un certain nombre choisit d'y englober des faubourgs, ainsi Saint-Denis ${ }^{13}$, Douai ${ }^{14}$, Rouen ${ }^{15}$ ou Strasbourg ${ }^{16}$.

Sur la rive gauche, les faubourgs Saint-Germain et Saint-Marcel restent sans protection, mais résistent eux aussi à la peste : ils existent toujours en 1360 puisque le prévôt des marchands ordonne leur incendie (avec celui de Notre-Dame-des-Champs) afin que les Anglais ne puissent s'y établir ${ }^{17}$, et en dépit de ces tribulations, ils subsistent durant toute la guerre de Cent Ans ${ }^{18}$. Ainsi, les archives seigneuriales du bourg Saint-Germain ne gardent pas trace des trous opérés par la peste - les cens continuent à être payés - ni des troubles des années 1356-1360. Le bourg est paradoxalement plus affecté par le renforcement de l'enceinte de Philippe Auguste et par la construction de l'enceinte de l'abbaye au début du règne de Charles V, qui conduisent à des démolitions. En revanche, il retrouve sa prospérité entre 1385 et 1418 lorsque beaucoup d'officiers royaux et de bourgeois parisiens enrichis s'y installent ${ }^{19}$. En 1500, les témoignages de voyageurs et les premiers plans de Paris montrent que les faubourgs de Paris se développent au-delà du rempart de Charles V rive droite, autour des rues Saint-Honoré, de Montmartre, Saint-Denis, Saint-Martin et Saint-Antoine ${ }^{20}$. Les faubourgs résistent donc à la crise, même s'il est probable que la densité de leur population ait chuté plus fortement que celle de la capitale.

Mais cet indice démographique est très imprécis, il faut en chercher d'autres susceptibles d'éclairer l'évolution économique sur le temps long. Autant la ville est pauvre en sources fiscales, autant elle est riche en sources foncières, car son sol est massivement

\footnotetext{
${ }^{10}$ Bernard Rouleau Paris, histoire d'un espace, Paris, Seuil, 1997, p. 165.

${ }^{11}$ Alain Salamagne, Construire au Moyen Âge, Les chantiers de fortification de Douai, Villeneuve d'Asq, PUS, p. 65-67.

${ }^{12}$ Nicolas Faucherre, «L'apport de l'enceinte à l'histoire des fortifications urbaines », dans Paul Van Ossel (sous la direction de), Les jardins du Carrousel, op. cit., p. 187-188, 196-197.

${ }^{13}$ Les bourgs de l'abbaye de Saint-Denis sont enclos dès 1356 dans une vaste enceinte qui inclut des espaces non-bâtis. Les constructions ne cessent pas dans la deuxième moitié du XIV siècle, si bien que le tissu urbain atteint alors sa plus grande densité (Michael Wyss (sous la direction de), Atlas historique de Saint-Denis, Paris, Maison des sciences de l'homme, 1996, p. 194-196, 227, 239).

${ }^{14}$ Alain Salamagne, Construire au Moyen Âge, op. cit., p. 65-67.

${ }^{15}$ François Neveux, «Les agglomérations castrales en Normandie (XI ${ }^{-}-\mathrm{XV}^{\mathrm{e}}$ siècles), dans André Chédeville et Daniel Pichot (sous la direction de), Des villes à l'ombre des châteaux, Rennes, PUR, 2010.

${ }^{16}$ Jean-Luc Pinol, Maurice Garden, Atlas historique des villes de France, Paris, Hachette, 1996, p. 124.

${ }^{17}$ Chronique dite de Jean de Venette, op. cit., p. 229.

${ }^{18}$ Journal d'un bourgeois de Paris, édité par Colette Beaune, Paris, Le livre de poche, 1990, p. 130, 225.

${ }^{19}$ Françoise Lehoux, Le bourg Saint-Germain-des-Prés depuis ses origines jusqu'à la fin de la Guerre de Cent ans, Paris, l'Auteur, 1951, p. 146-147.

${ }^{20}$ Clément Gurvil, Les paysans de Paris du milieu du XV siècle au début du XVIIe siècle, Paris, Champion, 2010, p. 40.
} 
possédé par des seigneurs ecclésiastiques dont les archives ont été bien conservées. Ces sources seigneuriales conservent la trace de diverses étapes du fonctionnement du marché immobilier qui peuvent être autant d'indices de la santé économique et démographique de la population, à défaut d'en donner une estimation exacte. On emprunte ici les séries chiffrées aux annexes de la thèse dactylographiée inédite de Simone Roux sur le marché immobilier de la rive gauche du XIII ${ }^{\mathrm{e}}$ au $\mathrm{XV}^{\mathrm{e}}$ siècle ${ }^{21}$.

La répartition chronologique des 1189 mentions de ventes conservées dans les fonds des seigneurs ecclésiastiques sur la période, essentiellement dans les registres d'ensaisinement seigneuriaux, permet de mesurer le dynamisme du marché immobilier (graphique 1). Bien qu'on en trouve trace dès 1209, ce genre d'information reste mal conservé jusqu'en 1350 : on n'en trouve qu'1 à 4 par an, avec pas mal d'années lacunaires. La principale cause en est que les registres d'ensaisinement de l'abbaye Saint-Geneviève, dont la seigneurie couvre la moitié de la rive gauche, ne commencent qu'à cette époque. Ils forment en revanche une série continue jusqu'en 1500 , et sont la principale source d'information disponible ${ }^{22}$. Il faut toutefois noter qu'ils présentent une lacune importante entre 1397 et 1408 , qui se traduit par une raréfaction de l'information à ce moment-là ${ }^{23}$. Ce brusque souci de conservation après 1350 est peut-être un effet de la crise, mais il révèle en même temps une forte activité foncière dans la seconde moitié du XIV ${ }^{\mathrm{e}}$ siècle, et spécialement entre 1350 et 1370 . A cette phase intense de transactions succède une chute brutale entre 1420 et 1431, puis une lente reprise jusqu'en 1480, avec un niveau d'activité inférieur à celui du XIV siècle. Le dynamisme du marché est donc vigoureux tout au long du XIV ${ }^{\mathrm{e}}$ siècle, mais il s'effondre avec la conquête anglaise en 1420.

\footnotetext{
${ }^{21}$ Simone Roux, Le quartier de l'université à Paris du XIII au XVe siècle : étude urbaine, thèse de doctorat d'Etat, Paris X, 1989, p. 1100-1126. On ne peut que se désoler que cet ouvrage majeur sur l'histoire économique de la capitale n'ait pas trouvé d'éditeur. Puisse cet article, qui y puise largement, rappeler sa valeur !

${ }^{22}$ AN, S* 1646 (1350-1356) à S* 1649 (1471-1500).

${ }^{23} \mathrm{La}$ lacune se trouve au sein du registre $\mathrm{S}^{*} 1647: 1^{\text {er }}$ cahier $(1359-1381), 3^{\text {ème }}$ cahier $(1381-1387), 4^{\text {ème }}$ cahier (1388-1397), $2^{\text {ème }}$ cahier (1408-1430). Vu la discordance chronologique des cahiers, il faut probablement imputer la perte du cahier concernant les années 1298-1407 au relieur.
} 


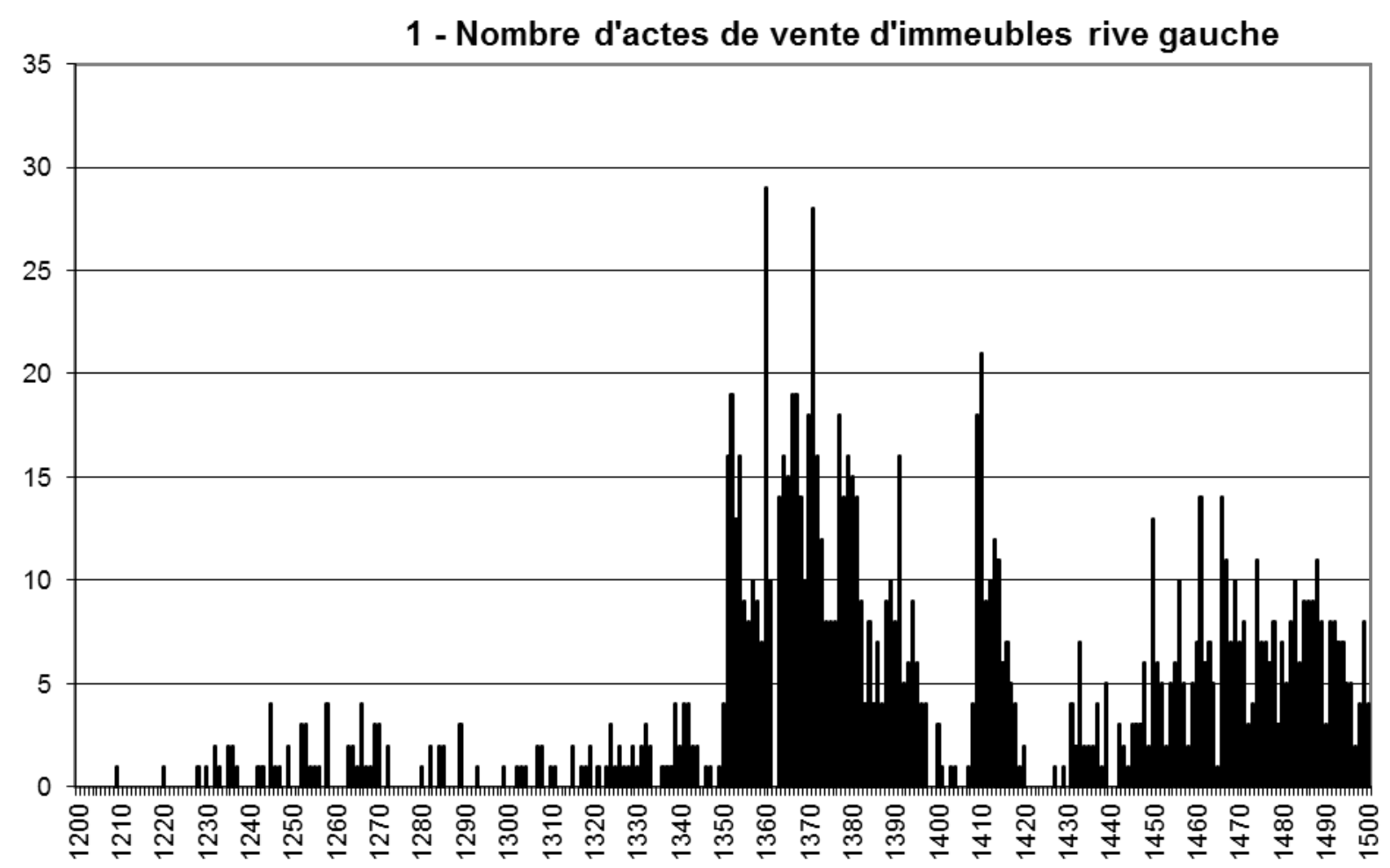

Les prix de vente des immeubles donnés dans ces actes offrent une évolution moins lisible, car ils portent sur des biens de nature très différente (graphique 2). Simon Roux note la bonne tenue des prix au XIV ${ }^{\mathrm{e}}$ siècle, en dépit d'un tassement dans les années 1366-1375, mais les exploite peu ${ }^{24}$. Les moyennes mobiles sur 10 ans et 30 ans (graphique 3), qui offrent l'avantage de lisser les écarts de prix, font plutôt ressortir une phase de plateau jusqu'en 1320 avec des prix de vente autour de $60 \mathrm{£p}$ en moyenne tridécennale, puis deux cycles d'inflation/déflation : hausse des prix entre 1320 et 1347 autour de $120 £$, puis chute jusqu'en 1370, suivie d'une nouvelle hausse jusqu'en 1420 au même niveau, puis chute jusqu'en 1440, suivie d'une troisième hausse jusqu'à $160 £$ vers $1500^{25}$. La peste entraîne une chute de la demande, donc des prix après 1347 , mais on notera un grand nombre de transactions foncières dans les années 1350-1380, qui sont peut-être l'effet de la réorganisation des patrimoines consécutive aux épidémies de 1348 et 1362, à moins qu'elles ne soient dynamisées par la sédentarisation de la cour à Paris à partir de Charles V. Quoiqu'il en soit, le marché résiste et retrouve un niveau de prix équivalent vers 1400. En revanche les prix et nombre de transactions s'effondrent avec l'occupation anglaise. Il peut paraître curieux que les prix ne soient pas plus bas en 1430 qu'en 1250, alors que tous les témoignages concordent pour décrire une ville ruinée, mais cela s'explique probablement par une inflation rampante que l'on peut observer sur le cours du marc d'argent tout au long du $\mathrm{XIV}^{\mathrm{e}}$ et du XV $\mathrm{XV}^{\mathrm{e}}$ siècle $^{26}$. Les prix confirment donc la bonne tenue relative du marché immobilier et la confiance des acteurs au XIV ${ }^{\mathrm{e}}$ siècle, en dépit des aléas de la conjoncture.

\footnotetext{
${ }^{24}$ Simone Roux, Le quartier de l'université, op. cit, p. 721-722.

${ }^{25}$ Les moyennes mobiles offrent l'avantage de lisser les données, mais perdent en précision chronologique du fait même qu'elles agrègent plusieurs années d'un coup, ce qui explique que leurs pics soient décalés selon qu'elles portent sur 10 ou 30 ans. Il faut donc s'appuyer sur l'évolution annuelle pour cerner la chronologie exacte des cycles.

${ }^{26}$ Marc Bompaire, Françoise Dumas, Numismatique médiévale, Turnhout, Brépols, 2000, annexe L.
} 
2 - Prix moyen des maisons rive gauche, en $£$ parisis

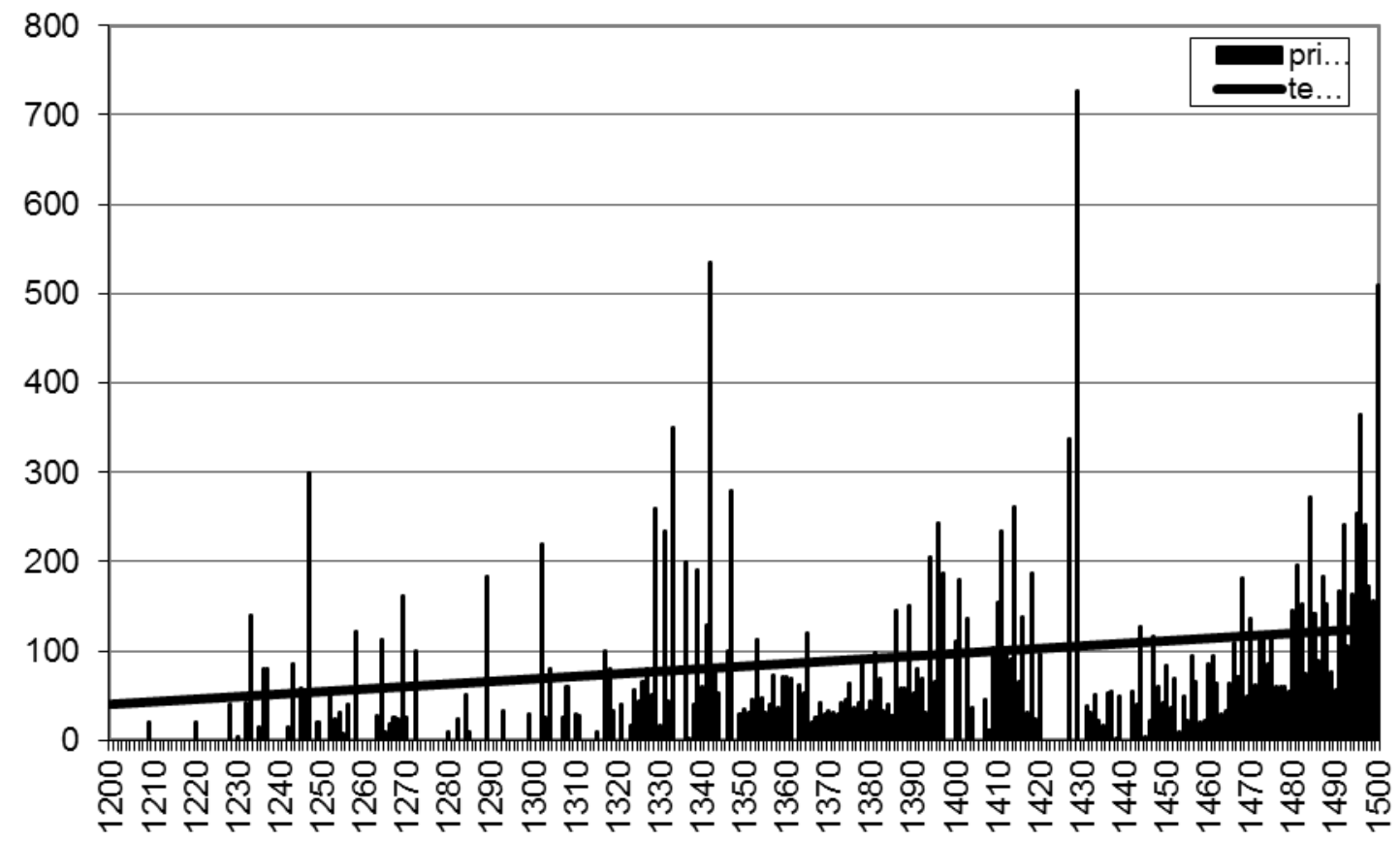

3 - Prix de vente moyens des immeubles rive gauche (en £p.)

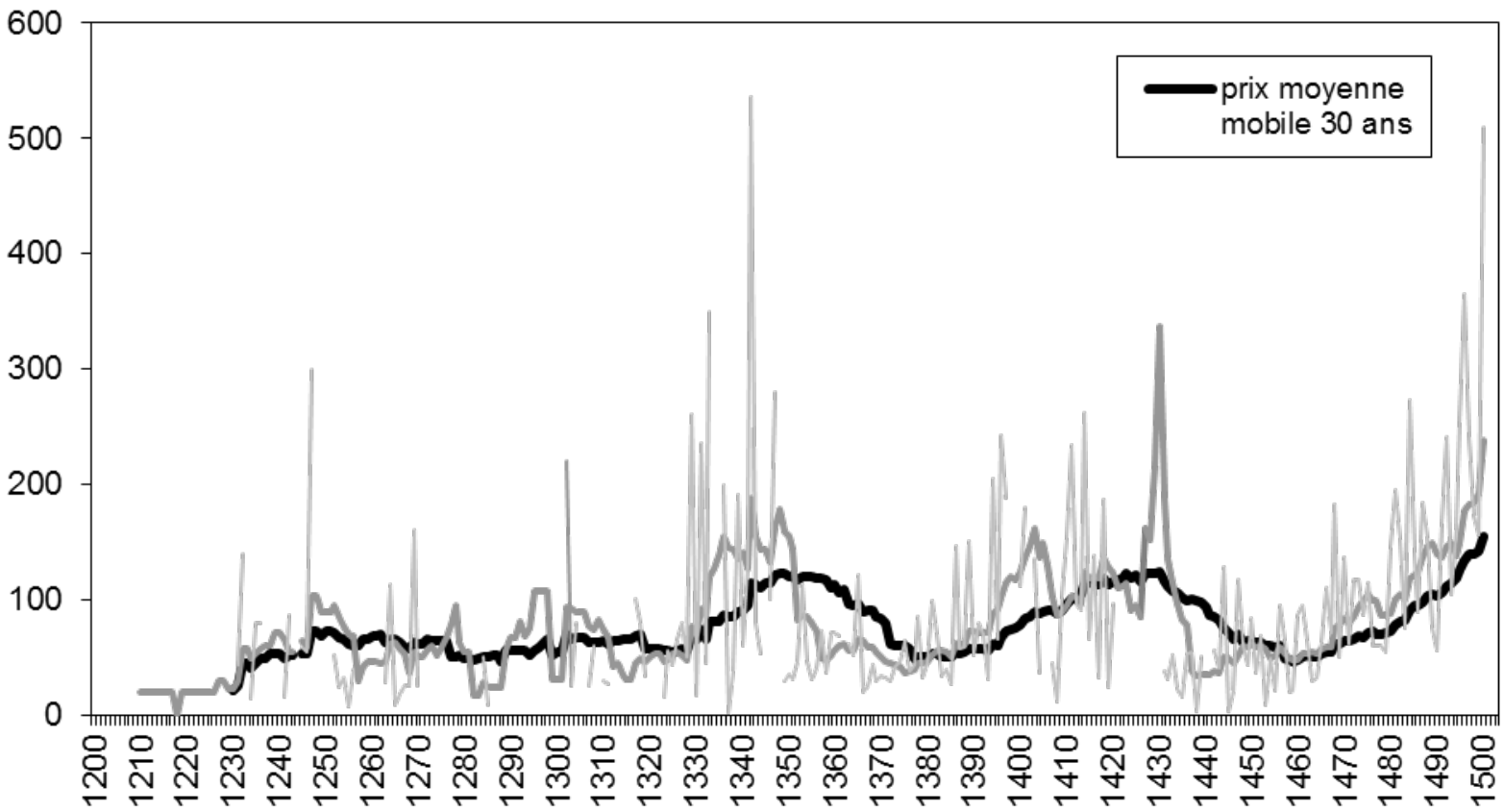

Plus significatifs d'une crise du marché immobilier sont les modérations et les mises en criées d'immeubles. Les modérations sont les réductions du montant des charges qui grèvent les immeubles. Il faut que la situation soit grave pour qu'un seigneur ecclésiastique accepte ce genre de compromis par lequel il renonce à une partie de son revenu pour se donner une chance de percevoir le reste - le risque s'il refusait serait que l'occupant de la maison quitte les lieux, et que les rentes restent impayées faute d'occupant. Or seulement 8 des 43 modérations relevées par Simone Roux se situent avant 1418 (graphique 4), le reste étant postérieur à cette date et se concentrant spécialement sur la période 1419-1461 (33 
modérations). Quant aux montants des modérations consenties (graphique 5), ils sont beaucoup plus forts au XV (40 £p) qu'au XIV ${ }^{\mathrm{e}}$ siècle $(16 £ p)$.

\section{4 - Nombre de modérations de rentes}

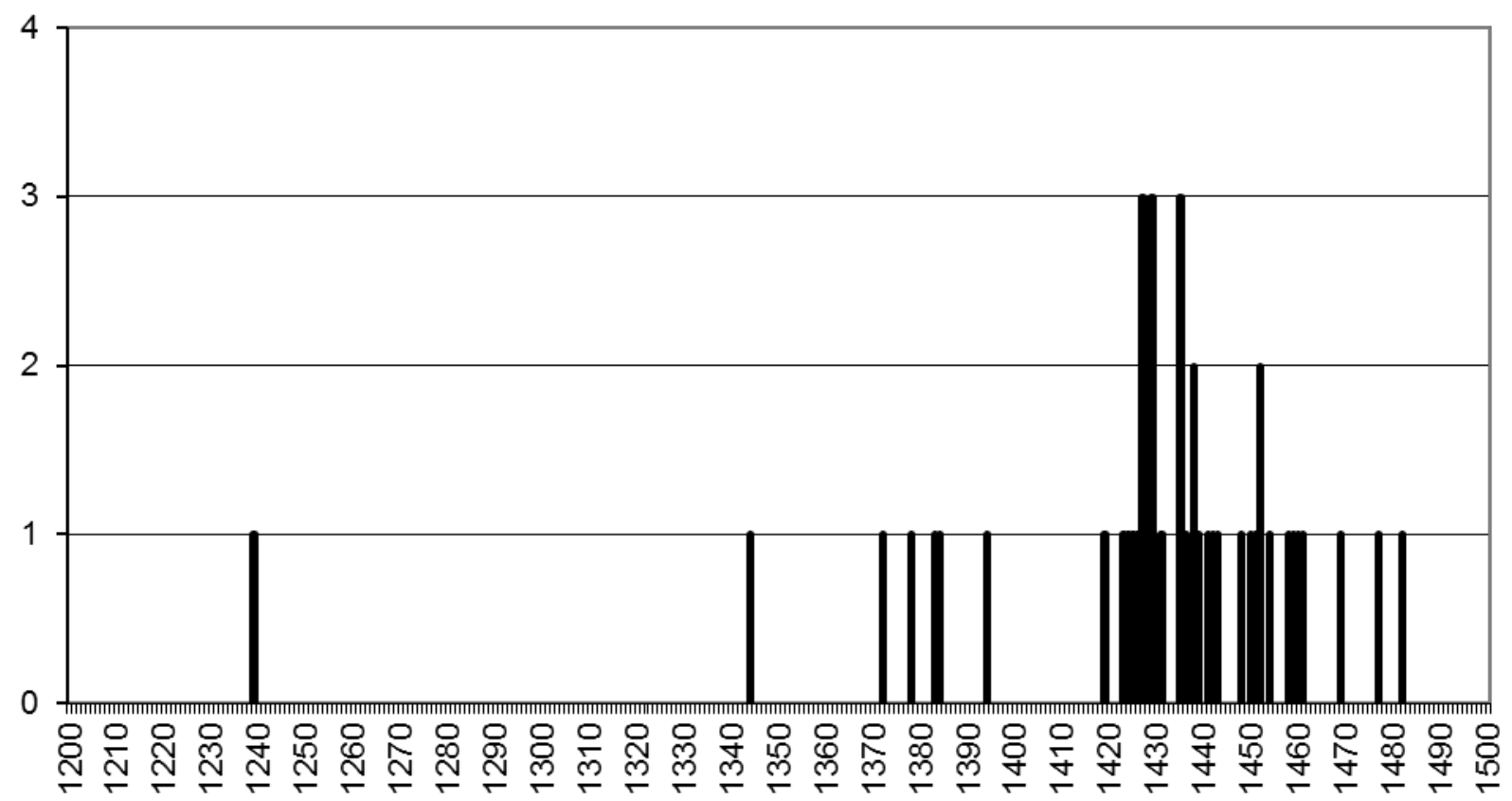

\section{5 - Pourcentage de modération des rentes}

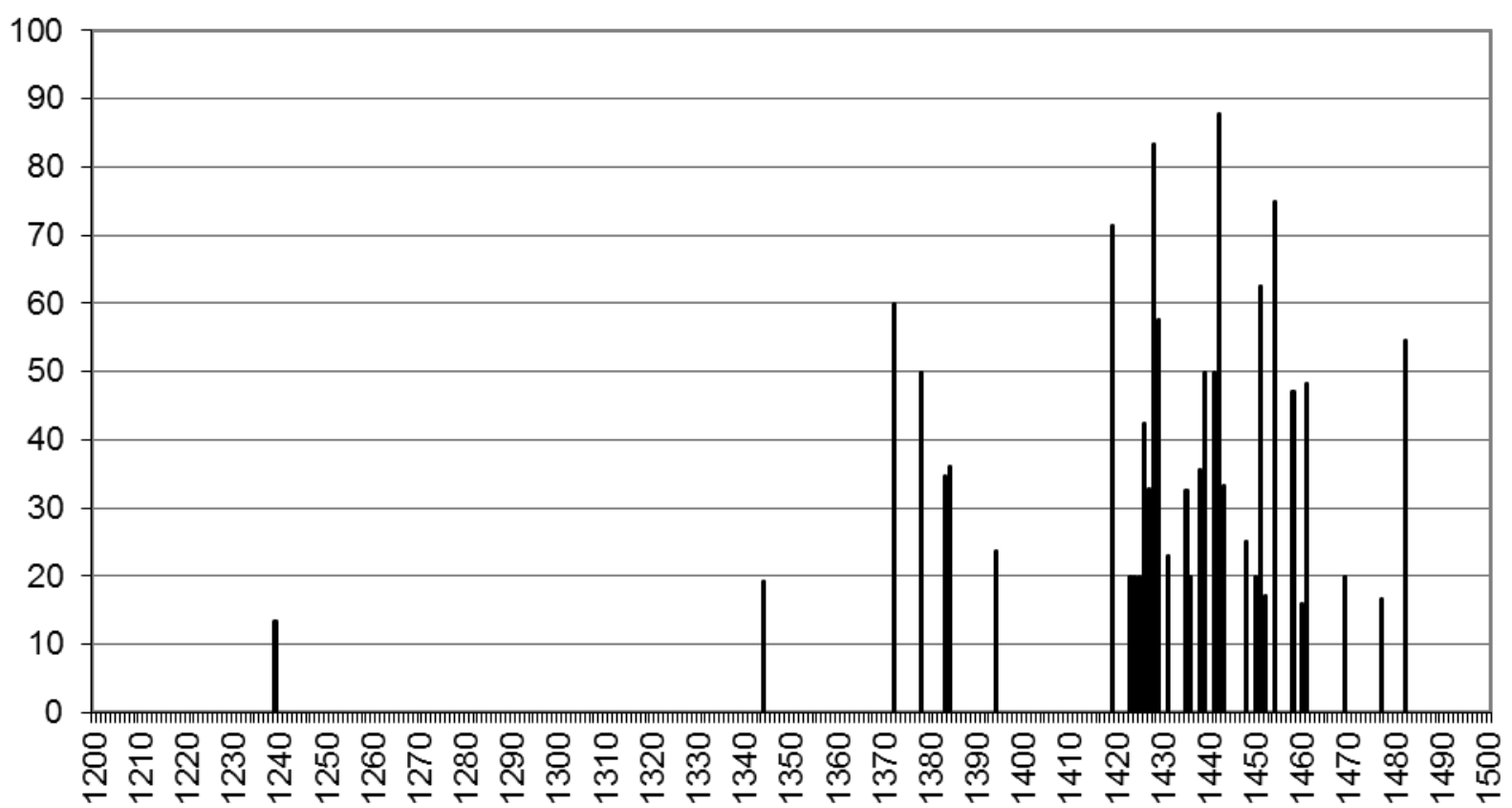

Mais les 123 mises en criées recensées forment peut-être l'indicateur le plus sûr de la crise immobilière (graphique 6). Elles procèdent du privilège, accordé aux bourgeois de Paris qui possèdent une rente sur une maison, d'assigner en justice le propriétaire qui cesserait de la payer : c'est la «criée » par un sergent de ville qui le somme de payer ou de déguerpir. La rente portant sur l'immeuble et non la personne, le propriétaire peut en effet choisir 
d'abandonner son bien ; il est alors tenu quitte du paiement de la rente et le seigneur se tourne vers le crédit-rentier dont la rente a été crée en dernier pour le sommer d'abandonner sa rente ou d'assumer la propriété de la maison et ses charges (il passe alors du statut de rentier à celui de tenancier). Comme le crédit-rentier qui a déguerpi a rarement payé ses arrérages, un rentier peut préférer abandonner sa rente plutôt que de solder les dettes de l'occupant précédent. Le seigneur se retourne alors contre le crédit-rentier dont la rente a été crée immédiatement avant celle qui vient d'être annulée, et recommence la procédure jusqu'à ce qu'un rentier assume la propriété de l'immeuble. Faute de quoi, l'immeuble lui revient en tant qu'ultime créancier, mais il est alors déchargé des rentes qui le grevaient, ce qui lui permet de trouver facilement un nouveau tenancier, auquel il enjoint en général d'investir une certaine somme pour réparer l'immeuble qui s'est entretemps dégradé ${ }^{27}$. Or la chronologie des criées recensées sur la rive gauche ne commence pas avant 1304 ; elles deviennent plus régulières à partir de 1376 mais s'intensifient entre 1419 et 1434 puisque près de la moitié des criées (52) se concentre sur ce faible intervalle, qui est aussi celui de l'occupation anglaise. La seigneurie de SainteGeneviève est représentative de la conjoncture économique parisienne, car des criées apparaissent au même moment dans les fonds seigneuriaux de la rive droite: elles se concentrent sur la période 1406-1432 pour le fief de Tiron, 1418-1454 pour Saint-Germaindes-Prés, 1420-1455 pour Saint-Merri, et 1438-1464 pour le Temple ${ }^{28}$.

\section{6 - Nombre de criées}

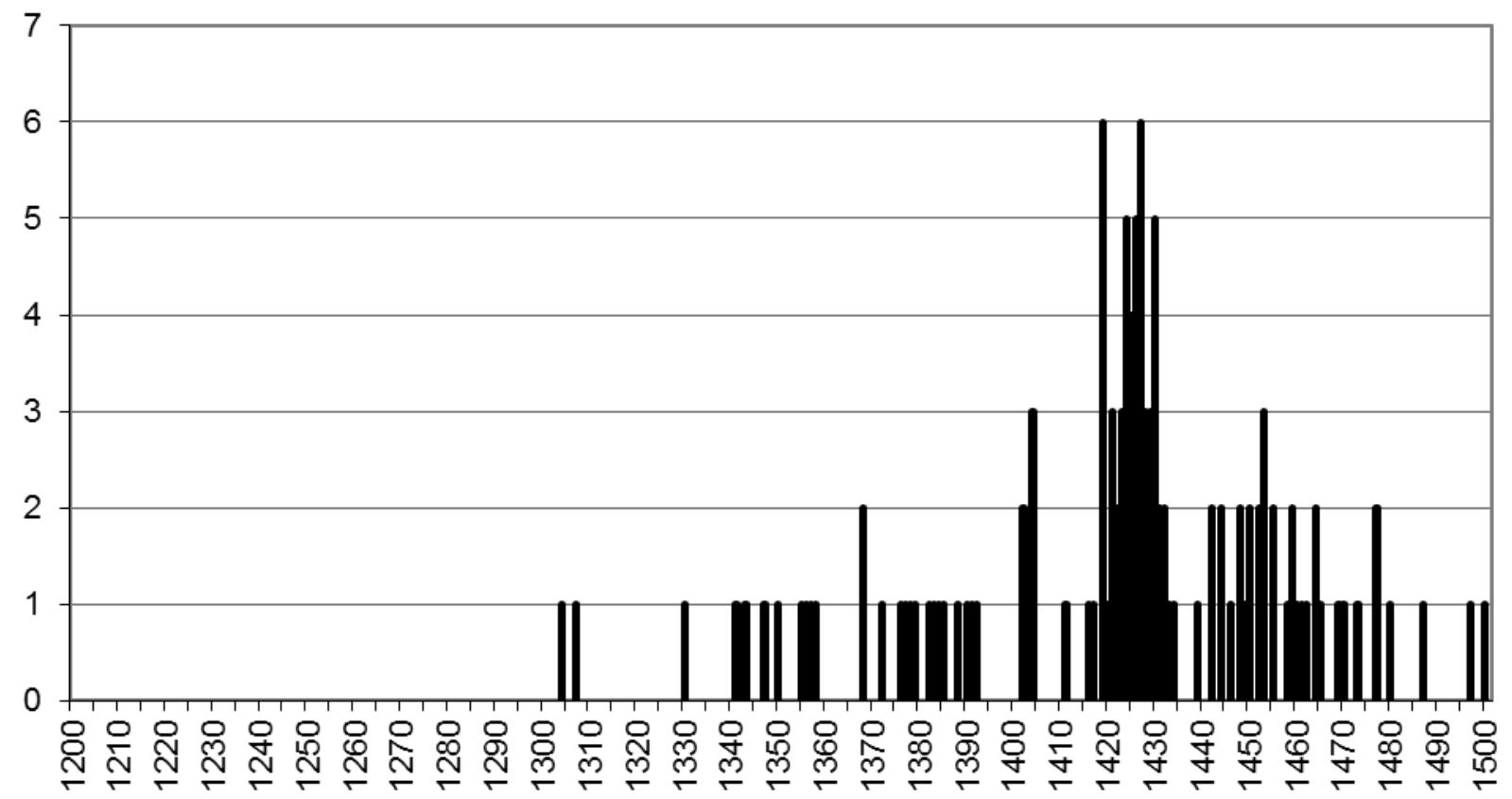

La chronologie de ces différents indicateurs est donc à peu près concordante : le XIII ${ }^{\mathrm{e}}$ siècle est mal renseigné, mais semble une période faste pour le marché immobilier, avec des prix stables, quelques rares modérations, mais le système fonctionne puisque les occupants des immeubles assument les charges qui les grèvent et ne suscitent pas de criées pour défaut de paiement de rentes. La ville atteint un sommet démographique dans les années 1330-1340,

\footnotetext{
${ }^{27}$ Sur le fonctionnement du marché immobilier parisien, voir Boris Bove, Benoît Descamps, Yvonne-Hélène Le Maresquier, Simone Roux, "Le marché immobilier à Paris (XIII $-\mathrm{XV}^{\mathrm{e}}$ siècles)», dans C. Bourlet (sous la direction de), Les prix et les salaires à Paris du XIII e au XVI siècle, à paraître sur http://aedilis.irht.cnrs.fr/paris/. ${ }^{28}$ Valentine Weiss, Cens et rentes à Paris au Moyen Age. Documents et méthodes de gestion domaniale, Paris, Champion, 2009, p. 1146.
} 
suscitant une hausse tendancielle des prix des maisons. Des sources enfin abondantes révèlent un marché foncier très actif dans la seconde moitié du XIV siècle, mais des prix de l'immobilier en baisse, ce qui s'explique probablement par un recul de la demande, lié à l'affaissement de la population de la ville. Quelques nuages apparaissent cependant avec l'apparition des criées au XIV ${ }^{\mathrm{e}}$ siècle, surtout après 1350 . Le marché immobilier est donc ébranlé, mais résiste bien comme le suggèrent les prix qui repartent à la hausse dès 1370 . Les indicateurs sont ensuite unanimes pour refléter un effondrement du marché immobilier entre 1420 et 1434, c'est-à-dire durant la période de domination anglaise. Que l'effondrement immobilier coïncide avec l'effondrement politique paraît cohérent ; ce qui l'est moins, c'est la résistance, voire la bonne santé du marché au XIV ${ }^{\mathrm{e}}$ siècle, alors que la crise économique est patente ailleurs depuis le début du siècle et que la crise politique bat son plein dans la capitale. On ne peut donc que noter une nette discordance entre le rythme de la crise immobilière à Paris et le modèle de la crise de la fin du Moyen Âge évoqué plus haut.

\section{Les causes du décalage entre crise générale et relative prospérité à Paris}

Si le marché immobilier ne s'effondre pas, c'est que la demande reste forte en dépit des aléas, donc que la population ne baisse pas trop et surtout reste solvable. Il y a plusieurs explications à cela.

D'abord le maintien d'une certaine immigration rurale, en dépit de l'effondrement démographique des campagnes, qui permet de combler en partie les brèches provoquées par les épidémies. Les campagnes franciliennes sont densément peuplées, mais en période d'insécurité, les fortifications de la capitale ont pu attirer nombre de laboureurs désireux de se mettre à l'abri, eux et leurs biens, des réquisitions des armées en campagnes, puis des forfaits des grandes compagnies. D'ailleurs, le phénomène ne concerne pas que les paysans, car une lettre d'Étienne Marcel aux échevins d'Ypres en 1358 évoque plus d'un millier de nobles venus trouver refuge dans la ville lors de la Jacquerie ${ }^{29}$. C'est d'ailleurs peut-être en prévision de ces paniques que le tracé de 1356 comprend volontairement de vastes espaces libres propres à accueillir hommes et bêtes fuyant les combats. Ces mouvements migratoires ponctuels ont aussi des effets structurels, car tous les paysans réfugiés ne repartent pas dans leur village après les troubles. Le phénomène est en tout cas patent au XVe siècle, avec l'apparition massive de « laboureurs » dans la population parisienne ${ }^{30}$.

L'autre facteur, et probablement le plus déterminant, réside dans l'attractivité que le statut de capitale donne à la ville. Paris est la capitale des rois de France depuis 1200, mais la cour demeurait itinérante ${ }^{31}$. L'avènement des Valois vient infléchir cette orientation : le roi Philippe VI de Valois qui monte sur le trône en 1328 est issu d'une branche cadette des Capétiens possessionnée en Île-de-France, ce qui la rend familière de Paris et encline à demeurer en Île-de-France. Ce changement dynastique est aussi l'occasion du déclenchement de la guerre de Cent Ans qui fixe les souverains dans la ville, tant pour s'y protéger que pour y préparer la défense du royaume. Charles V (1364-1380), son petit-fils, investit beaucoup d'argent et d'attention dans de grands chantiers de construction parisiens ou franciliens : outre l'achèvement du rempart et de la Bastille, il transforme le Louvre en palais, il fait construire l'hôtel Saint-Paul, bâtir Vincennes et rénover de nombreuses demeures dans les environs

\footnotetext{
${ }^{29}$ Roland Delachenal, Histoire de Charles V, Paris, Picard, 1909, I, p. 427.

${ }^{30}$ Simone Roux, Le quartier de l'université, op. cit., p. 644-645 et Clément Gurvil, Les paysans de Paris, op. cit., 2010, p. 112-113.

${ }^{31}$ Ainsi, en dépit du développement de l'appareil administratif dans Paris sous le règne de Philippe le Bel (12851314), le roi ne réside que 44 jours par an en moyenne dans la capitale, avec de forts contrastes selon les années (Elisabeth Lalou, Itinéraire de Philippe le Bel (1285-1314), Paris, De Boccard, 2007, p. 120, n. 315)
} 
immédiats de la capitale ${ }^{32}$. Son fils Charles VI est un roi presque totalement parisien durant tout son règne, de 1380 à 1422, comme le suggèrent les lieux de naissance de ses enfants : 9 sont nés à Paris et 3 dans ses environs (Beauté, Saint-Ouen, Melun) ${ }^{33}$.

Les troubles politiques n'ont donc pas que des effets pervers sur la vie urbaine : la concentration du mécontentement populaire est compensée par la présence prolongée, sinon permanente, du roi dans la ville ou à proximité, ainsi que l'afflux des richesses du royaume, ponctionnées à l'occasion de la guerre à travers la mise en place d'un impôt permanent à partir de 1369. Jusque là en effet, le roi devait vivre de ses propres ressources patrimoniales, ce qui limitait considérablement ses ambitions politiques; la rançon du roi Jean permit cependant de lever à partir de 1360 dans un cadre coutumier un impôt public régulier, qui se mua en impôt de guerre à partir de 1369. A partir de 1370, le roi obtint des pays de Languedoïl qu'ils paient chaque année l'impôt royal pour financer la reconquête des territoires cédés aux Anglais lors de la première partie de la guerre de Cent ans, sans se réunir pour négocier son principe ou son montant. En Languedoc, les états se réunirent tous les ans, mais acceptèrent de payer systématiquement l'impôt de guerre, puisque chasser les Anglais et les routiers semblait la seule solution pour rétablir la paix dans le royaume. La concentration des richesses entre les mains du souverain pour faire la guerre aux Anglais ne l'empêcha nullement d'en soustraire une partie pour financer une vie de cour brillante. Ses bâtiments sont l'expression la plus visible de cette politique de prestige, mais elle s'épanouit aussi dans une vie de cour animée ${ }^{34}$. Ces folles dépenses parisiennes sont perçues par les contemporains, qui en font grief à la monarchie lorsque vient la crise, la vraie, avec l'occupation anglaise ${ }^{35}$.

Mais le roi n'est donc pas seul à habiter la ville ; il attire autour de lui les élites du royaume, qui espère obtenir faveurs, argent et titres en venant à la cour. Le recensement des hôtels connus à Paris en 1400 est à ce titre éclairant, puisqu'on peut dénombrer 123 propriétés appartenant à la noblesse, moyenne et haute, ainsi qu'à des prélats. Chaque prince est entouré d'une vaste maisonnée dont les membres les plus éminents ont leur propre Hôtel (ainsi Charles de Savoisy, chambellan du duc Louis d'Orléans). Or l'entourage de ces personnages est très important : 700 personnes pour Charles VI, 250 pour Jean de Berry, 250 pour Louis d'Orléans, etc. si bien que Claude Gauvard a pu estimer à 10000 le nombre d'habitants de la ville appartenant officiellement à un $\mathrm{Hôtel}^{36}$. A ce personnel curial, il faut aussi ajouter les quelques centaines d'officiers des institutions centrales - Parlement, chambre des comptes, cour des aides, chambre du Trésor, etc. - qui se recrutent dans la noblesse et l'élite bourgeoise du royaume. Le poids de ce personnel dans l'économie parisienne n'est pas négligeable, car il représente $22 \%$ des acteurs du marché immobilier de la rive gauche au début du $\mathrm{XV}^{\mathrm{e}}$ siècle ${ }^{37}$. Le roi, les princes et les gens de cour qui l'entourent vivent à Paris et contribuent ainsi à soutenir la demande en biens de consommation ordinaires et de luxe, ce qui explique probablement la bonne résistance de l'économie parisienne en ces temps troublés.

Sur le plan strictement immobilier, on pourrait ajouter que ces propriétés sont vastes, car elles servent à accueillir la suite nombreuse des princes qui les possèdent, si bien qu'elles représentent $13 \%$ de la surface intra muros en 1400 (carte 2$)^{38}$. Les princes et leur suite contribuent ainsi à soutenir les prix immobiliers dans la ville. Il est vrai que les hôtels des princes laïcs sont plus vastes et que ceux-ci habitent plus volontiers la rive droite, tandis que les prélats affectionnent la rive gauche, mais il y a de brillantes exceptions, tel l'hôtel de

\footnotetext{
${ }^{32}$ Françoise Autrand, Charles V, Paris, Fayard, 1994, chap. XXXI.

${ }^{33}$ Ead., Charles VI, Paris, Fayard, 1992, p. 609

${ }^{34}$ Voir par exemple Paris 1400. Les arts sous Charles VI, Paris, Fayard-RMN, 2004.

35 Alain Chartier s'en fait l'écho en 1422 dans son Quadriloge invectif (traduction et édition par Florence Bouchet, Paris, Champion, 2002, p. 96).

${ }^{36}$ Claude Gauvard, Violence et ordre public au Moyen Âge, Paris, Picard, 2005, p. 227-244.

${ }^{37}$ Simone Roux, Le quartier de l'université, op. cit., p. 604.

${ }^{38} \mathrm{La}$ cartographie des hôtels princiers est disponible sur http://alpage.tge-adonis.fr/.
} 
Nesle. Surtout, les hôtels se répartissent équitablement entre les deux rives, par conséquent, le poids de la présence curiale se fait sentir aussi sur les statistiques foncières rive gauche qui servent ici d'indicateur. En effet, si on observe les variations des prix des maisons vendues plus de $100 \mathrm{fp}$ sur la rive gauche (la moyenne des prix de vente étant de $90 £ \mathrm{p}$ ), on observe qu'ils suivent les cycles observés plus haut, mais ne cessent de battre des records entre 1200 et 1427 (graphique 7).

\section{7 - Prix de vente supérieurs à $100 £ p$}

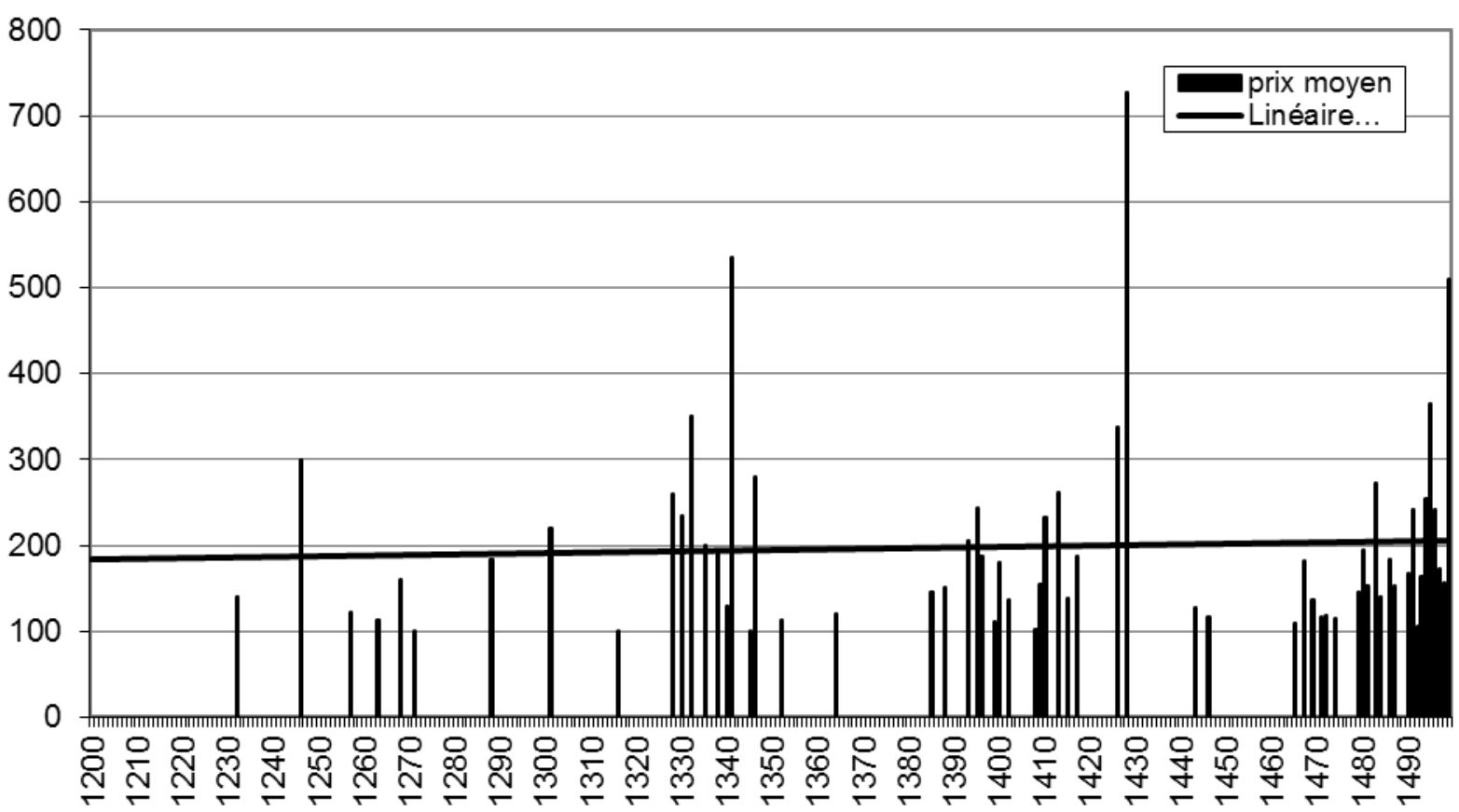

La fonction de capitale ne suscite donc pas que des révoltes à Paris, elle lui permet de maintenir un relatif dynamisme économique dans un contexte par ailleurs très perturbé. La preuve par l'absurde en est donnée dans la première moitié du $\mathrm{XV}^{\mathrm{e}}$ siècle lorsqu'elle perd ce rôle.

\section{L'effondrement du marché immobilier et la crise financière au $X V^{e}$ siècle}

La bonne tenue des prix depuis le XIII ${ }^{\mathrm{e}}$ siècle, qui se confirme au XIV ${ }^{\mathrm{e}}$ siècle, rend les Parisiens confiants dans le marché foncier et les conduit à se servir de leur patrimoine immobilier pour obtenir des liquidités. En cela, la crise immobilière entraîne une crise financière. En l'absence d'un crédit officiel licite, la constitution de rente est en effet le moyen le plus simple d'obtenir de l'argent. Elle consiste pour un propriétaire à créer une rente perpétuelle, gagée sur sa maison, qu'il s'engage à payer aux quatre termes accoutumés. Il reçoit donc un capital, moyennant le paiement tous les ans d'une somme qui est en général fixée à environ $10 \%$ du montant obtenu. En cas de défaut de paiement, le rentier fait «crier» la maison pour en obtenir la propriété.

La valeur de rentes est proportionnelle à celle de la maison, aussi tant que les prix de l'immobilier montent, elles peuvent s'accumuler sur un même immeuble au fil du temps. Elles peuvent même dépasser sa valeur réelle si les crédit-rentiers estiment le débit-rentier solvable, que celui-ci ait une activité économique profitable ou que les prêteurs aient confiance dans la solidité du marché immobilier parisien qui semble capable de résister aux 
secousses que la conjoncture lui impose. C'est ce qui s'est passé dans la seconde moitié du $\mathrm{XIV}^{\mathrm{e}}$ siècle durant laquelle tous les acteurs du marché semblent tabler sur la hausse de celuici : " tous estiment qu'on retrouvera la croissance et donc pensent qu'on peut continuer à vendre des rentes, à escompter des profits en hausse dans l'exploitation du capital immobilier. Il y a une sorte d'optimisme, jamais exprimé directement, mais à coup sûr présent, car nul n'a vraiment envisagé le blocage du système $»^{39}$. Il eut pourtant lieu en 1420 .

Le marché immobilier reposait sur un équilibre entre le coût de l'immeuble (c'est-àdire son prix d'achat ainsi que le poids de ses charges) et le profit escompté (c'est-à-dire son prix de vente ou son usage lucratif, qu'il soit commercial ou locatif). Or la conjoncture politique contribue à affaiblir l'économie parisienne :

- La guerre civile entre Armagnacs et Bourguignons a conduit à des purges sévères dans l'élite parisienne. Ainsi en 1413, les émeutes soutenues par le duc de Bourgogne Jean sans Peur pour faire pression sur les états généraux réunis à Paris conduisent à l'exécution de plusieurs dizaines de personnalités armagnacs. Quand les excès des émeutiers eurent retourné l'opinion en leur défaveur, les Armagnacs prirent le pouvoir dans Paris et procédèrent à une épuration anti-bourguignonne. L'impopularité $d u$ gouvernement armagnac incapable de faire face à la conquête anglaise conduisit les Parisiens à ouvrir leurs portes aux troupes bourguignonnes 1418, qui prirent alors leur revanche sur le personnel armagnac.

- La conquête anglaise en 1420 a conduit nombre de Parisiens, surtout parmi les notables, à fuir la ville pour suivre Charles VII dans son exil au sud de la Loire. On peut suivre l'hémorragie des riches parisiens dans les comptes des confiscations de biens dans la ville, chaque changement de tutelle politique s'accompagnant de la fuite des opposants et de la distribution de leurs maisons aux fidèles ${ }^{40}$.

- La guerre sans front en Île-de-France que mènent les Armagnacs-légitimistes aux Anglo-bourguignons dans les années qui suivent crée un climat de terreur et perturbe le commerce ainsi que le ravitaillement de la ville. C'est le moment où famines et épidémies semblent s'acharner sur la ville, comme en témoigne le Journal d'un bourgeois de Paris ${ }^{41}$;

- durant l'occupation anglaise, Paris perd son rôle de capitale au profit de Rouen pour la France anglaise, ou Tours pour la France delphinale.

Il en résulte un effondrement démographique et économique difficile à chiffrer, mais qui se traduit par la crise du marché immobilier : les propriétaires ne paient plus les charges des immeubles, qu'ils soient morts, en exil, ruinés ou qu'ils estiment que les charges sont trop lourdes pour le profit qu'ils attendent de l'immeuble. Ils cessent alors de ces entretenir, voire de payer les charges, d'où le recours croissant aux procédures de criée. Elles n'empêchent pas cependant la dégradation du parc immobilier, car les seigneurs attendent que les arrérages s'accumulent pour faire crier une maison. En outre, la procédure se déroule sur trois ans en moyenne : il y a trois criées séparées d'au moins 40 jours et il y a parfois des dizaines de crédi-rentiers dont il faut recueillir la volonté au sujet de leur rente (faire opposition à la criée ou abandonner le droit à percevoir la rente), sans compter les procès qui peuvent ralentir le processus. Or les maisons parisiennes sont avant tout bâties en bois et en plâtre, si bien qu'elles nécessitent un entretien permanent. Elles ont donc eu tout le temps de tomber en ruine lorsque la procédure de criée arrive à son terme : le seigneur peine alors à la bailler à rente un nouveau tenancier qui devra procéder à des réparations ruineuses.

\footnotetext{
${ }^{39}$ Simone Roux, Le quartier de l'université, op. cit., p. 723

${ }^{40}$ Les confiscations opérées sous le règne d'Henri V concernent 562 à 689 immeubles dans la ville (Guy Llewelyn Thompson, Paris and its Poeple under English Rule. The Anglo-Burgundian Regime, 1420-1436, Oxford, Clarendon press, 1991, p. 123-124).

${ }^{41}$ Journal d'un bourgeois de Paris, op. cit., passim.
} 
L'effondrement des prix de l'immobilier est donc étroitement lié à une crise financière. La détérioration de l'économie régionale et l'effacement de la vie de cour entraînent une crise économique et l'appauvrissement des propriétaires qui ne peuvent plus payer les charges de leur maison, ni obtenir du crédit en les hypothéquant. Le défaut de paiement des charges ruine les rentiers, qui ne sont autres que les citadins aisés et les églises, tandis que l'atonie du marché foncier ruine les seigneurs (surtout ecclésiastiques) qui ne touchent plus de droits de mutation. Pour parler en termes contemporains, l'effondrement des prix de l'immobilier entraîne l'éclatement d'une bulle spéculative fondée sur l'espérance d'une hausse infinie des prix fonciers, puis un krach économique, car le crédit reposait largement sur ces hypothèques.

\section{La lente sortie de crise dans la seconde moitié du XVe siècle}

La restauration de l'équilibre du marché tient à deux facteurs dont le poids respectif est difficile à mesurer : la restauration de la paix en Île-de-France en 1436 et dans le royaume en 1453 qui permet à l'économie de se redresser d'une part, et une réforme des règles régissant le marché immobilier d'autre part. On s'intéressera ici surtout au second facteur, plus précoce et dont le mécanisme est plus facile à cerner.

Les seigneurs ont été impuissants à enrayer la crise immobilière, car ils ne se sont résolus à accepter des modérations de rente ou des remises d'arrérages que tardivement et avec parcimonie - d'où le faible nombre de modérations recensées (43) par rapport aux criées (123). Ces seigneurs ecclésiastiques étaient en effet très attachés à l'inaliénabilité de leurs droits, fussent-ils stériles.

L'initiative revint donc au roi qui modifia progressivement, en tâtonnant, les règles du marché immobilier à partir de 1424 par une série d'ordonnances ${ }^{42}$. Il est motivé par le souci de restauration matérielle de la capitale, désormais remplies de maisons "vides et vagues ». Son action consiste surtout :

- à permettre aux propriétaires de racheter quand ils le souhaitent les rentes qui grevaient leur maison à un taux fixé à 12 fois le montant annuel de la rente ;

- à limiter le poids des charges à $1 / 3$ du prix de la maison, ce qui suppose que sa valeur marchande soit estimée par des experts ;

- à accélérer la procédure de mise en criée d'une maison : elle doit débuter dès l'absence de paiement de rente constatée. Les crédit-rentiers doivent saisir la justice immédiatement sous peine de perdre leurs droits si c'est le commissaire du roi qui déclenche la procédure.

Cette réforme consiste finalement à donner plus de latitude aux propriétaires face à leur seigneur pour retrouver un équilibre financier, ce qui allait contre les habitudes seigneuriales, mais aussi contre la gestion routinière des établissements ecclésiastiques dont une grande partie des revenus consistaient en rentes données par les fidèles. Ils durent cependant plier devant la volonté royale d'assainir le marché immobilier, donc l'économie de la capitale.

La crise du marché immobilier n'est qu'un aspect de la crise économique, mais les prix fonciers étant le seul indicateur observable précisément sur le temps long, ils paraissent un bon point de vue sur la crise des $\mathrm{XIV}^{\mathrm{e}}$ et $\mathrm{XV}^{\mathrm{e}}$ siècles à Paris. Leur étude fait ressortir plusieurs traits rarement mis en avant par l'historiographie de la crise de la fin du Moyen Âge. D'abord la discordance des rythmes entre crise nationale et crise locale : la crise ne frappe durement la ville qu'après 1420. Cette discordance s'explique par le statut de capitale et de ville de cour de Paris, mais aussi par les effets d'aubaine suscités par la crise : afflux de

\footnotetext{
${ }^{42}$ Simone Roux, Le quartier de l'université, op. cit., p. 895-915.
} 
population attirée par la sécurité des remparts, sédentarisation des rois à Paris, concentration des richesses dans la capitale avec la mise en place de l'impôt permanent. La crise de la fin du Moyen Âge a ses limites, qu'il conviendrait maintenant d'explorer. Ensuite, les rythmes de la crise locale semblent parfaitement en phase avec ceux de la politique, puisqu'ils sont concomitants de la guerre civile et de l'occupation anglaise, ce qui réhabilite le facteur politique comme cause profonde de ces évolutions économiques, tant pour la croissance que pour la dépression. La crise financière des années 1420 est une conséquence de la crise immobilière, mais cette dernière procède de la guerre civile et de la conquête anglaise qui privent la ville de ses plus riches citadins et désorganise les circuits économiques dans la France du nord. Dans ce cas, les facteurs économiques sont loin d'être au cœur du mécanisme de la crise de l'économie urbaine.

\section{Résumé}

L'historiographie des années 1970-1980 s'est beaucoup intéressée à «la crise de la fin du Moyen Âge » et l'a envisagée comme une crise systémique qui frappait tous les secteurs de la vie humaine et aboutissait même à une crise de la civilisation. Mais à trop vouloir donner une unité dépressive aux $\mathrm{XIV}^{\mathrm{e}}$ et $\mathrm{XV}^{\mathrm{e}}$ siècles, ce courant a négligé d'observer les différences de rythme de cette crise selon les années, les lieux et les secteurs, de même que les effets d'aubaine qu'elle peut aussi porter en elle. Envisager la crise à l'échelle locale permet de cerner les rythmes discordants entre crise générale et crise locale. Le cas parisien est un observatoire privilégié puisque son statut de capitale place la ville au cœur de la crise politicoéconomique de la fin du Moyen Âge, tandis que d'abondantes sources foncières permettent de suivre assez précisément les prix de l'immobilier qui paraît être un bon indicateur de la réalité de la crise. Cela permet de montrer que la crise générale frappe la capitale de manière différente selon les époques et peut même parfois lui profiter, en particulier au XIV ${ }^{\mathrm{e}}$ siècle.

Historiography of 70-80's theorised the crisis of the end of the Middle Age. It proposed a systemic interpretation of all phenomenon of this period, which are all connected in the vicious circle of a great depression. Trouble is that the Parisian economy seems to resist quite well in the XIV ${ }^{\text {th }}$ century, although political trouble are numerous after 1356. Prices of real estates give an idea of the health of the local economy despite the Black Death and Hundred years war. So the real estate market is under stress during the $\mathrm{XIV}^{\text {th }}$ century but resists quite well to the general crisis, whereas it collapsed after 1420 . The reason is that the general crisis provides some positive effects in the capital: kings remain in Paris war obliges them to organize defence from the capital; war obliged kings to raise taxes, but a part of remained in their hands for courtly life; a brilliant court attracts lords who settled too in the capital: they owned $13 \%$ of the land of the city and their household was about 10000 people. This context supports prices and entertains confidence in the local economy. 
Illustrations

- Carte de Paris vers 1380 (PDF)

- Carte des hôtels aristocratiques en 1400 (PDF)

- Graphique 1 - Nombre d'actes de vente d'immeubles rive gauche

- Graphique 2 - Prix moyen des maisons rive gauche, en $£$ parisis

- Graphique 3 - Prix de vente moyens des immeubles rive gauche (en fp.)

- Graphique 4 - Nombre de modérations de rentes

- Graphique 5 - Pourcentage de modération des rentes

- Graphique 6 - Nombre de criées

- Graphique 7 - Prix de vente supérieurs à $100 \mathrm{fp}$ 
